\title{
DESENVOLVIMENTO DE MUDAS DE Swietenia macrophylla EM RESPOSTA A NITROGÊNIO, FÓSFORO E POTÁSSIO
}

\author{
Carlos Alberto Franco Tucci ${ }^{1}$, José Zilton Lopes Santos ${ }^{1}$, Clauzio Heitor da Silva Júnior ${ }^{2}$, \\ Patrícia Aparecida de Souza ${ }^{3}$, Iza Maria Paiva Batista ${ }^{4}$, Nelson Venturin ${ }^{5}$ \\ ${ }^{1}$ Eng. Agrônomo, Dr., Depto. Engenharia Agrícola e Solos, UFAM, Manaus, AM, Brasil - ctucci@ufam.edu.br; \\ ziltonlopes@ufam.edu.br \\ ${ }^{2}$ Zootecnista, M.Sc, UFAM, Manaus, AM, Brasil - clauziohsj@terra.com.br \\ ${ }^{3}$ Eng $^{\mathrm{a}}$ Florestal, Dr ${ }^{\mathrm{a}}$., Fundação Universidade Federal do Tocantins, UFT, Gurupi, TO, Brasil - patriciaapsouza@ yahoo.com.br \\ ${ }^{4}$ Lic. em Ciências Agrárias, M.Sc., UFAM, Manaus, AM, Brasil - im_paiva@ @otmail.com \\ ${ }_{5}^{5}$ Eng. Florestal, Dr., UFLA, Lavras, MG, Brasil - venturim@dcf.ufla.br \\ Recebido para publicação: 05/05/2010 - Aceito para publicação: 19/04/2011
}

\begin{abstract}
Resumo
Objetivou-se avaliar neste estudo o efeito de nitrogênio, fósforo e potássio no crescimento de mudas de mogno. As plantas foram cultivadas num Latossolo Amarelo Distrófico argiloso com seis níveis de nitrogênio $\left(0,50,100,125,150\right.$ e $\left.200 \mathrm{~kg} \mathrm{~N} \mathrm{ha}^{-1}\right)$, seis níveis de fósforo $(0,75,150,225,300$ e $400 \mathrm{~kg}$ $\mathrm{P}_{2} \mathrm{O}_{5}$ ha $\left.^{-1}\right)$ e seis níveis de potássio $\left(0,40,80,120,160\right.$ e $\left.200 \mathrm{~kg} \mathrm{~K} \mathrm{ha}^{-1}\right)$, em condição de casa de vegetação. Aos 100 dias após o transplantio, as plantas foram colhidas para determinar as características de crescimento: altura, diâmetro, matéria seca da parte aérea e raízes e a concentração de nutrientes presentes na parte aérea e raízes. O desenvolvimento das mudas de mogno é pouco influenciado pelo fornecimento de doses crescentes dos nutrientes nitrogênio, fósforo e potássio, indicando uma baixa exigência nutricional da espécie na fase inicial de crescimento. Entre os nutrientes avaliados, a espécie parece ligeiramente mais exigente quanto ao potássio em relação aos demais. No entanto, a demanda da planta já seria satisfeita com uma pequena adição do respectivo nutriente. A adubação com nitrogênio e fósforo não promove melhora na qualidade das mudas de mogno.
\end{abstract}

Palavras-chave: Espécies florestais; mogno; nutrição mineral; crescimento inicial.

\begin{abstract}
Development of Swietenia macrophylla seedlings in response to nitrogen, phosphorus and potassium. A Greenhouse experiment was developed in order to investigate effects of nitrogen, phosphorus and potassium on the growth of mahogany seedlings (Swietenia macrophylla King). The plants were cultured in clayey dystrophic Red Latosol containing six nitrogen levels $\left(0,50,100,125,150\right.$ and $\left.200 \mathrm{~kg} \mathrm{~N}^{-1}\right)$, six phosphorus levels $\left(0,75,150,225,300\right.$ and $400 \mathrm{~kg} \mathrm{P}_{2} \mathrm{O}_{5}$ ha $\left.^{-1}\right)$ and six potassium levels $(0,40,80,120,160$ and $\left.200 \mathrm{~kg} \mathrm{~K} \mathrm{ha}^{-1}\right)$. After 100 days of transplanting, the plants were harvest and processed for determine height, diameter, dry weight and nutrient concentration in roots and shoots. Addition of nitrogen, phosphorus and potassium did not affect the mahogany seedlings development. In contrast to $\mathrm{N}$ and $\mathrm{P}$, the yield of plants was slightly greater with $\mathrm{K}$ fertilization. In general, results of this study clearly demonstrate the absence of a beneficial effect of these nutrients on the growth of mahogany seedlings. The nitrogen and phosphorus did not improve the quality of mahogany seedlings.
\end{abstract}

Keywords: Forest species; mahogany; mineral nutrition; early growth.

\section{INTRODUÇÃO}

A Swietenia macrophylla King, pertencente à família Meliaceae, conhecida vulgarmente por mogno, ocorre em toda a região amazônica, sendo mais frequente na região sul do Pará (LORENZI, 1998). A importância da exploração do mogno deve-se ao seu alto valor econômico no mercado internacional de madeiras tropicais, sendo uma das espécies mais exploradas no Brasil, no entanto, ameaçada de extinção, principalmente por não haver renovação dos estoques através de reflorestamento (SANTOS, et al., 2008). Um dos maiores entraves à implantação de plantios puros ou mistos com essa espécie se deve à falta de estudos com essa espécie. 
Diante disso, conhecimentos relativos ao seu manejo necessitam ser adquiridos, entre os quais aqueles relacionados à produção de mudas para fins de reflorestamento (CORDEIRO et al., 2009). Essa preocupação se deve ao fato de que um dos fatores de maior importância na implantação de qualquer cultura é a utilização de mudas de boa qualidade, pois essa qualidade se reflete diretamente na maior taxa de sobrevivências das mudas e na maior produção na fase jovem, assim como no maior potencial de produção na fase adulta. Segundo Dias et al. (2006), o bom desenvolvimento das mudas ocorrerá quando o solo for fértil e apresentar concentrações adequadas principalmente de nitrogênio $(\mathrm{N})$, fósforo $(\mathrm{P})$ e potássio $(\mathrm{K})$, além de outros elementos que são indispensáveis para a saúde e vigor das mudas, como manganês, enxofre, ferro, cobre, zinco, cloro e boro. A falta de estudos envolvendo a absorção de nutrientes e os requerimentos nutricionais dessas espécies florestais nativas tem se constituído num dos maiores entraves para o seu uso em plantios comerciais ou na recuperação de áreas degradadas (BERNADINO et al., 2005).

Essa espécie é característica do ecossistema de terra firme, onde predominam Latossolos Amarelos e Argissolos, solos altamente intemperizados, com fortes limitações nutricionais (FALCÃO; SILVA, 2004), sugerindo que ela apresenta uma boa adaptação a solos pouco férteis (LAMBERS; POORTER, 1992; CRAVO; SMYTH, 1992), sendo ainda classificada como espécie clímax (LORENZI, 1998). Resultados de outros estudos (SILVA et al., 2007; SANTOS, et al., 2008; WALLAU et al., 2008; TUCCI et al., 2009) mostram respostas positivas a doses crescentes de calcário, fósforo (P), potássio (K), cobre $(\mathrm{Cu})$ e nitrogênio $(\mathrm{N})$ em todas as características de crescimento na fase de produção de mudas em condições de casa de vegetação, indicando o potencial de crescimento rápido dessa espécie em condições favoráveis, como relatado por Lamprecht (1990).

Portanto, levando-se em conta que nos últimos anos os projetos de reflorestamento com espécies de alto valor comercial como o mogno têm se tornado cada vez mais comuns na região amazônica brasileira, bem como o fato de haver evidências de que a qualidade das mudas dessa espécie pode ser influenciada pela nutrição mineral, a compreensão básica sobre os fatores que afetam as taxas de desenvolvimento da planta, como N, P e K, implicaria um manejo mais racional, mediante a aplicação de significativas quantidades de fertilizantes. Isso sem contar que a adubação onera sobremaneira a implantação de espécies florestais, devendo, portanto, ser bem manejada em todos os aspectos.

Objetivou-se, no presente estudo, avaliar o comportamento de mudas de mogno quanto ao crescimento vegetativo inicial, em resposta a doses de N, P e K, em condições de casa de vegetação. Este trabalho se justifica em função de haver evidências de que a qualidade das mudas dessa espécie pode ser influenciada pela nutrição mineral com NPK.

\section{MATERIAL E MÉTODOS}

Foi conduzido um ensaio em casa de vegetação, no Departamento de Engenharia Agrícola e Solos da Universidade Federal do Amazonas (UFAM), utilizando-se amostra da camada subsuperficial (20-40 cm de profundidade) de um Latossolo Amarelo Distrófico argiloso, coletado no Setor Sul do Campus da UFAM, cujas coordenadas em UTM são: 21M016894, 9657166.

Antes da instalação dos experimentos, o material foi seco ao ar e passado em peneira de $4 \mathrm{~mm}$ de abertura. Subamostras do solo foram passadas em peneiras de $2 \mathrm{~mm}$ e submetidas a análises químicas, cujos resultados são apresentados na tabela 1 .

Tabela 1. Principais atributos químicos do Latossolo Amarelo Distrófico (20 - $40 \mathrm{~cm}$ de profundidade) usado no experimento.

Table 1. Main chemical attributes of dystrophic Red Latosol (20 - $40 \mathrm{~cm}$ depth) used in the experiment.

\begin{tabular}{|c|c|c|c|c|c|c|c|c|c|c|c|}
\hline \multirow{2}{*}{$\mathrm{pH}\left(\mathrm{H}_{2} \mathrm{O}\right)$} & $\mathbf{P}$ & $\mathbf{K}$ & $\mathrm{Ca}$ & Mg & Al & $\mathrm{H}+\mathrm{Al}$ & $\mathbf{t}$ & $\mathbf{T}$ & V & $\mathbf{m}$ & MO \\
\hline & \multicolumn{2}{|c|}{ mg.dm ${ }^{-3}$} & $\ldots$ & ....... & $10 I_{c} d$ & -..-.-. & 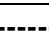 & & $--(\%)$ & & g.kg-1 $^{-1}$ \\
\hline 4,3 & 1,0 & 24,0 & 0,1 & 0,1 & 1,2 & 6,3 & 1,5 & 6,6 & 4,0 & 82,0 & 18,2 \\
\hline
\end{tabular}

Foram conduzidos três experimentos, para adubação nitrogenada, fosfatada e potássica, respectivamente. As plantas foram cultivadas em sacos plásticos de polietileno com capacidade para $4,0 \mathrm{dm}^{3}$. O substrato recebeu calcário dolomítico com PRNT $=100 \%$, com dosagem equivalente a 2 ton.ha ${ }^{-1}$. Em seguida, os solos foram incubados por 30 dias. Após o período de incubação do calcário, 
em todas as parcelas, efetuou-se a aplicação dos tratamentos e uma adubação básica com macro e micronutrientes.

Para cada experimento, os tratamentos foram dispostos em delineamento inteiramente casualizado, com seis doses e três repetições. Cada unidade experimental foi constituída por quatro plantas. Os tratamentos foram constituídos por seis doses de $\mathrm{N}\left(0,50,100,125,150\right.$ e $\left.200 \mathrm{~kg} \mathrm{~N} \mathrm{ha}^{-1}\right)$ na forma de ureia, seis doses de $\mathrm{P}\left(0,75,150,225,300\right.$ e $400 \mathrm{~kg} \mathrm{P}_{2} \mathrm{O}_{5}$ ha $\left.^{-1}\right)$ na forma de superfosfato triplo e seis doses de $\mathrm{K}\left(0,40,80,120,160\right.$ e $\left.200 \mathrm{~kg} \mathrm{~K} \mathrm{ha}^{-1}\right)$ na forma de cloreto de potássio.

A adubação básica foi composta de $100,300,200,30$ e $50 \mathrm{~kg}$ de N, $\mathrm{P}_{2} \mathrm{O}_{5}, \mathrm{~K}_{2} \mathrm{O}$, S e FTR$\mathrm{BR} 12 . \mathrm{ha}^{-1}$. Essa adubação foi feita utilizando-se as fontes ureia, superfosfato triplo, cloreto de potássio, sulfato de amônio e FTR-BR12, sendo a aplicação da ureia, do cloreto de potássio e do superfosfato triplo feito na forma de solução. No caso do superfosfato triplo, ele foi agitado com água num liquidificador, visando uma melhor solubilização da fonte. Por outro lado, a fonte de micronutriente FTR-BR12 foi fornecida na forma de pó. No caso da solução, esta continha todos os nutrientes, exceto aquele que estava sendo testado no respectivo experimento. $\mathrm{O}$ fornecimento do $\mathrm{N}$ foi feito de forma parcelada, em três aplicações com intervalos de 15 dias, para todos os experimentos. Após a adubação do substrato e da aplicação dos tratamentos, estes foram umedecidos por 10 dias com $500 \mathrm{ml}$ de água deionizada.

Quanto à obtenção das mudas, visando acelerar a germinação das sementes, estas foram imersas em água por 24 horas, posteriormente tratadas com o fungicida Benlate na concentração de $0,1 \%$ e postas para germinar em areia lavada. Após a emergência das plântulas e formação de dois pares de folhas, procedeu-se ao transplantio. Durante o período experimental, a umidade do solo foi mantida em torno de $60 \%$ do VTP (FREIRE et al., 1980), por meio de irrigações diárias com água deionizada.

Aos 100 dias após o transplantio, foram avaliados a altura da parte aérea e o diâmetro do colo. Nessa mesma época, as plantas foram colhidas e foram avaliados o comprimento de raízes e o material vegetal (raízes e parte aérea), que foi posto a secar em estufa de circulação forçada de ar a $70{ }^{\circ} \mathrm{C}$ até peso constante, para determinação da matéria seca da parte aérea (MSPA) e raízes (MSR), relação raiz/parte aérea (R/PA), além do IQD - índice de qualidade de Dickson (DICKSON et al., 1960). O material da parte aérea foi moído e submetido à análise, conforme Malavolta et al. (1997), para determinação dos teores de nutrientes. Com base nos teores dos elementos avaliados e na produção de matéria seca, foi calculado o acúmulo do nutriente.

Os dados obtidos foram submetidos à análise de variância e testes de médias (Scott Knott, 5\%), para se avaliar a diferença entre tratamentos, utilizando-se o programa Sistema para Análises Estatísticas(SAEG) versão 9.1 (SAEG, 2007).

\section{RESULTADOS E DISCUSSÃO}

\section{Adubação nitrogenada}

De modo geral, o fornecimento de doses crescentes de $\mathrm{N}$ não proporcionou ganhos significativos em nenhuma das variáveis avaliadas (altura, diâmetro, MSPA, MSR e R/PA) (Tabela 2). A ausência de ganhos significativos em crescimento evidencia a baixa responsividade dessa espécie à adubação nitrogenada na fase de muda. Ao contrário das espécies pioneiras, que têm seu potencial de crescimento reduzido quando se desenvolve em solos pobres, o grupo ecológico clímax ao qual pertence a espécie mogno tem o estímulo ao crescimento proporcionado pela adubação menos pronunciado e algumas vezes inexistente, o que é atribuído em parte ao crescimento mais lento das espécies pertencentes a esse grupo. No entanto, no caso do presente estudo, essa ausência de resposta pode ser em função de a baixa exigência da espécie ser suprida apenas com o $\mathrm{N}$ presente na matéria orgânica do solo, já que, apesar dos teores estarem na classe baixa, conforme Comissão de Fertilidade do Solo do Estado de Minas Gerais (CFSMG, 1999), a sua mineralização seria suficiente para manter o crescimento inicial da planta. A isso somam-se as reservas de N presentes na semente. Por outro lado, Lambers e Poorter (1992) relatam que espécies de crescimento lento apresentam baixa resposta ao fornecimento de nutrientes, característica, em alguns casos, relacionada à adaptação a solos pouco férteis. Porém Tucci et al. (2009), avaliando o efeito de doses crescentes de $\mathrm{N}$ na produção de mudas de mogno num Latossolo Amarelo Distrófico típico, com doses bastante similares às utilizadas no presente estudo, observaram resposta a dose moderada de $\mathrm{N}$ (57,5 g de $\mathrm{N}$ ton. ${ }^{-1}$ de substrato, o equivalente a $125 \mathrm{~kg} \mathrm{~N}$ ha $^{-1}$ no presente estudo).

De forma semelhante, porém em condições de solução nutritiva, Wallau et al. (2008) verificaram redução na produção de massa seca total de mudas de mogno, especialmente na omissão de $\mathrm{N}$, indicando 
maior demanda desse nutriente na fase inicial de desenvolvimento da planta, o que não foi observado no presente estudo.

Tabela 2. Resumo das análises de variância para as variáveis altura da parte aérea, diâmetro do colo, matéria seca da parte aérea (MSPA), matéria seca da raiz (MSR) e relação raiz/parte aérea (R/PA) das mudas de mogno, em resposta a doses de nitrogênio.

Table 2. Summary of analysis of variance of variables shoot height, diameter, shoot dry matter (MSPA), root dry matter (MSR) and the root/shoot (R/PA) of mahogany seedlings in response to nitrogen doses.

\begin{tabular}{lcccccc}
\hline \multirow{2}{*}{ Fontes de variação } & \multirow{2}{*}{ G.L. } & \multicolumn{5}{c}{ Q.M. } \\
\cline { 3 - 7 } & & Altura & Diâmetro & MSPA & MSR & R/PA \\
\hline Blocos & 2 & 0,443 & 0,508 & 0,899 & 1,718 & 1,625 \\
Tratamento & 5 & $0,356^{\text {ns }}$ & $2,139^{\text {ns }}$ & $2,120^{\text {ns }}$ & $2,169^{\text {ns }}$ & $2,333^{\text {ns }}$ \\
Resíduo & 10 & 69,664 & 1,753 & 9,248 & 0,420 & $0,565 \mathrm{E}^{-2}$ \\
C.V. $(\%)$ & & 10,0 & 6,6 & 14,2 & 13,4 & 10,4 \\
\hline
\end{tabular}

ns: não significativo ao nível de 5\%; Q.M.: quadrado médio.

Respostas avaliadas no presente trabalho quanto ao conteúdo de nutrientes na parte aérea de mudas do mogno, em função da adição de diferentes doses de $\mathrm{N}$ no substrato, permitem melhor discriminar o padrão de resposta da espécie estudada em relação ao fornecimento do nutriente. Notou-se que, apesar dos teores dos nutrientes $\mathrm{N}, \mathrm{S}$ e $\mathrm{Cu}$ na parte aérea da planta serem influenciados de maneira pouco consistente pelas doses de $\mathrm{N}$ no substrato (dados não apresentados), o conteúdo de nenhum macro ou micronutriente foi influenciado pela adição de doses crescentes de N. Baixa resposta à adição de nutrientes em espécies florestais nativas de crescimento lento, na fase inicial de crescimento, foi observada por Lima et al. (1997), em estudo no qual a altura, diâmetro da copa e da base das plantas foi favorecido apenas pela dose de $25 \mathrm{~kg}$ de $\mathrm{N}$ por hectare. Esse comportamento corrobora os encontrados no presente estudo, confirmando uma tendência geral de baixa capacidade de absorção de $\mathrm{N}$ por espécies classificadas como clímax (GONÇALVES, 1992).

\section{Adubação fosfatada}

De maneira semelhante ao comportamento observado para o $\mathrm{N}$, a adição de doses crescentes de $\mathrm{P}$ ao substrato não contribuíram de forma significativa para o aumento dos parâmetros biométricos testados nas mudas de mogno, como altura, diâmetro, MSPA, MSR e R/PA (Tabela 3), mesmo em um solo com teor de P disponível de $1 \mathrm{mg} \cdot \mathrm{dm}^{-3}$ (Tabela 1 ).

Tabela 3. Resumo das análises de variância para as variáveis altura da parte aérea, diâmetro do colo, matéria seca da parte aérea (MSPA), matéria seca da raiz (MSR) e relação raiz/parte aérea (R/PA) das mudas de mogno, em resposta a doses de fósforo.

Table 3. Summary of analysis of variance of variables shoot height, diameter, shoot dry matter (MSPA), root dry matter (MSR) and the root/shoot (R/PA) of mahogany seedlings in response to phosphorus doses.

\begin{tabular}{lcccccc}
\hline \multirow{2}{*}{ Fontes de variação } & \multirow{2}{*}{ G.L. } & \multicolumn{5}{c}{ Q.M. } \\
\cline { 3 - 7 } & 2 & Altura & Diâmetro & MSPA & MSR & R/PA \\
\hline Blocos & 2,323 & 1,262 & 1,537 & 0,265 & 0,280 \\
Tratamento & 5 & $1,586^{\text {ns }}$ & $1,937^{\text {ns }}$ & $1,203^{\text {ns }}$ & $0,442^{\text {ns }}$ & $0,835^{\text {ns }}$ \\
Resíduo & 10 & 56,529 & 1,518 & 3,874 & 0,287 & $0,111 \mathrm{E}^{-1}$ \\
C.V $(\%)$ & & 10,8 & 9,5 & 19,6 & 28,3 & 17,6 \\
\hline
\end{tabular}

ns: não significativo ao nível de 5\%; Q.M.: quadrado médio.

Os resultados confirmam o baixo potencial de resposta da espécie à adição desse nutriente nessa fase de crescimento. Wallau et al. (2008), avaliando sintomas de deficiências nutricionais em mudas de mogno em solução nutritiva, verificaram que na omissão de $\mathrm{P}$ obteve-se uma pequena redução no crescimento. Ressalta ainda que, nessa condição, não foram observados sintomas característicos de deficiência desse nutriente, o que confirma uma possível formação de um estoque e a utilização eficiente 
do mesmo pelas plantas. Relatos de ausência de resposta de algumas espécies florestais, tais como eucalipto (MAFFEIS et al., 2000) e castanheira-do-brasil (CAMARGOS et al., 2002), ao fornecimento de $\mathrm{P}$ em solução nutritiva tem sido comum. No entanto, tal comportamento possivelmente se deve ao fato de fornecimento de $\mathrm{P}$ em quantidade suficiente durante a fase de adaptação das plantas com solução nutritiva completa, o que poderia contribuir para formação de uma reserva, de modo a não apresentar sintomas característicos de deficiência. Além disso, o requerimento externo de $\mathrm{P}$ pode também estar associado a diversos fatores, como tamanho e conteúdo de nutrientes das sementes, grau de desenvolvimento do sistema radicular, dependência micorrízica, taxa de crescimento e estádio de desenvolvimento da planta. No caso do mogno, a semente pode conter uma reserva de $\mathrm{P}$ (P-fitina) que pode fornecer quantidade suficiente desse nutriente na fase inicial de desenvolvimento das plantas. Santos et al. (2008), avaliando o desenvolvimento de espécies florestais de diferentes grupos sucessionais em função da adição de doses crescentes de $\mathrm{P}$, verificaram que as espécies climácicas guanandi e óleo-bálsamo praticamente não tiveram alteração do crescimento em função do aumento da dose de $\mathrm{P}$, comprovando que, apesar do $\mathrm{P}$ ser considerado um nutriente extremamente limitante ao crescimento inicial e estabelecimento da maioria das espécies cultivadas, principalmente para aquelas com sistema radicular pouco desenvolvido. No caso das espécies florestais nativas de crescimento lento, uma proposta que parece condizente com as informações até aqui obtidas é a de que essas plantas apresentam baixa eficiência de utilização, sendo também menos responsivas ao fornecimento de nutrientes, entre os quais o $\mathrm{P}$.

De maneira semelhante, Lima et al. (1997), avaliando o comportamento das mudas de espécies climácicas em campo, observaram que elas não apresentaram resposta ao fornecimento de $\mathrm{P}$ aos oito meses pós-plantio. Essa diferença de comportamento das espécies climácicas em relação às pioneiras se deve, em parte, ao fato de as pioneiras apresentarem sistema radicular mais desenvolvido e maior densidade de raízes finas, o que propicia taxas de crescimento e de absorção de nutrientes mais elevadas (GONÇALVES et al., 1992). No entanto, resposta positiva à adição de $\mathrm{P}$ em mudas de mogno, tanto para doses moderadas (RESENDE et al., 1999) quanto para maiores doses (SANTOS et al., 2008), tem sido observada.

Essa baixa capacidade de resposta da espécie mogno ao fornecimento de $\mathrm{P}$ na fase de muda ficou bastante evidente quando foram analisados os teores e conteúdos foliares dos demais nutrientes (dados não apresentados). De maneira bastante análoga, porém menos significativa, em relação ao comportamento mostrado para o fornecimento de $\mathrm{N}$, apenas o teor foliar de $\mathrm{Zn}$ e o conteúdo de $\mathrm{P}$ na parte aérea foram afetados pelas doses de $\mathrm{P}$ adicionadas ao substrato. Quanto aos teores, verificaram-se menores valores de $\mathrm{Zn}$ na parte aérea a partir da segunda dose $\left(75 \mathrm{~kg} \mathrm{P}_{2} \mathrm{O}_{5} \mathrm{ha}^{-1}\right)$, fato que possivelmente está relacionado com a interação negativa entre os dois elementos (CARNEIRO et al., 2008). Já os conteúdos de $\mathrm{P}$ na parte aérea tenderam a aumentar com adição de doses crescentes, porém tanto as três primeiras doses quanto as três ultimas não diferiram estatisticamente entre si.

\section{Adubação potássica}

A adição de K no solo não afetou de forma significativa o desenvolvimento de mudas de mogno quanto aos parâmetros biométricos (Tabela 4), exceto a MSPA (Tabela 5).

Tabela 4. Resumo das análises de variância para as variáveis altura da parte aérea, diâmetro do colo, matéria seca da parte aérea (MSPA), matéria seca da raiz (MSR) e relação raiz/parte aérea (R/PA) das mudas de mogno, em resposta a doses de potássio.

Table 4. Summary of analysis of variance of variables shoot height, diameter, shoot dry matter (MSPA), root dry matter (MSR) and the root/shoot (R/PA) of mahogany seedlings in response to potassium doses.

\begin{tabular}{lcccccc}
\hline \multirow{2}{*}{ Fontes de variação } & \multirow{2}{*}{ G.L. } & Altura & Diâmetro & QSPA & MSR & R/PA \\
\cline { 3 - 7 } & 2 & 3,109 & 0,874 & 0,708 & 1,108 & 0,897 \\
Blocos & 5 & $3,006^{\text {ns }}$ & $3,318^{\text {ns }}$ & $15,548^{*}$ & $1,258^{\text {ns }}$ & $2,307^{\text {ns }}$ \\
Tratamento & 10 & 25,430 & 2,834 & 4,615 & 1,081 & $0,678 \mathrm{E}^{-1}$ \\
Resíduo & & 6,3 & 10,5 & 13,0 & 26,2 & 32,2 \\
C.V $(\%)$ & &
\end{tabular}

*: Significativo; ns: não significativo ao nível de 5\%; Q.M.: quadrado médio. 
De acordo com os resultados, verifica-se que, apesar da produção de MSPA ser influenciada pela adição de doses crescentes de $\mathrm{K}$, esse efeito foi verificado apenas para a segunda dose $\left(40 \mathrm{~kg} \mathrm{~K}_{2} \mathrm{O} \mathrm{ha}^{-1}\right)$ quando comparado à ausência de fornecimento de K (testemunha) (Tabela 5). Essas observações sugerem que em condições nas quais a disponibilidade de $\mathrm{K}$ é limitada (Tabela 1), apenas a adição de $40 \mathrm{~kg} \mathrm{~K} \mathrm{~K}_{2} \mathrm{O}$ $\mathrm{ha}^{-1}$ já é suficiente para a espécie atingir a máxima produção, indicando que a espécie tem sua demanda atendida mesmo em condições de baixa disponibilidade de $\mathrm{K}$ no solo, ou, ainda, que a mesma é bem eficiente em converter esse nutriente em biomassa quando a sua disponibilidade é limitada.

Tabela 5. Matéria seca da parte aérea (MSPA) de mudas de mogno, em resposta a doses de potássio, aos 100 dias em condição de casa de vegetação.

Table 5. Shoot dry matter (MSPA) of mahogany seedlings in response to potassium doses, after 100 days in greenhouse condition.

\begin{tabular}{lc}
\hline $\begin{array}{l}\text { Doses de } \mathbf{K}_{\mathbf{2}} \mathbf{O} \\
\text { kg.ha }^{-1}\end{array}$ & $\begin{array}{c}\text { MSPA } \\
\text { g/unidade experimental }\end{array}$ \\
\hline 0 & $2,74 \mathrm{~b}$ \\
40 & $6,03 \mathrm{a}$ \\
80 & $6,02 \mathrm{a}$ \\
120 & $5,85 \mathrm{a}$ \\
160 & $6,70 \mathrm{a}$ \\
200 & $5,89 \mathrm{a}$ \\
\hline Médias seguidas de mesmas letras não diferem estatisticamente ao nível de 5\%.
\end{tabular}

Comportamento semelhante de espécies clímax quanto ao fornecimento de doses crescentes de $\mathrm{K}$ foi observado por Silva et al. (1997). Esses autores, avaliando diferentes espécies, tanto pioneiras quanto clímax, verificaram que estas últimas se equipararam em comportamento, e a produção de matéria seca de raiz, diâmetro do caule e altura foi pouco influenciada pela adição desse nutriente, levando a concluir que na fase de mudas as espécies clímax possuem um baixo requerimento de $\mathrm{K}$, ou se mostram eficientes em utilizar esse nutriente em condições de baixa disponibilidade. No entanto, a ausência de resposta do mogno ao fornecimento de $\mathrm{K}$ parece estar relacionada ao baixo requerimento de $\mathrm{K}$ nessa fase de crescimento. Segundo Silva et al. (1997), os efeitos relativos ao potássio são muito pequenos, inexistentes e até mesmo depressivos para o crescimento de certas espécies florestais nativas. Por outro lado, resultados contrastantes sobre o requerimento de K pelo mogno são encontrados na literatura. Wallau et $a l$. (2008) observaram que em condição de solução nutritiva houve uma tendência de que a omissão de K proporcionasse uma redução na produção de massa seca total, indicando uma certa demanda desse nutriente na fase inicial de desenvolvimento da planta.

Em relação ao efeito da adição de doses crescentes de $\mathrm{K}$ no conteúdo dos nutrientes $\mathrm{N}, \mathrm{P}, \mathrm{K}, \mathrm{Ca}$, $\mathrm{S}, \mathrm{B}, \mathrm{Cu}, \mathrm{Mn}$ e $\mathrm{Zn}$ na parte aérea de mudas de mogno, nota-se que, ao contrário dos resultados observados para o fornecimento de diferentes doses de $\mathrm{N}$ e $\mathrm{P}$, houve efeito significativo para o conteúdo de todos os nutrientes avaliados, exceto o ferro (Tabela 6). De acordo com os resultados, verifica-se que, apesar de haver influência das doses de $\mathrm{K}$ nos conteúdos, o aumento desses valores foi observado apenas até a segunda dose $\left(40 \mathrm{~kg} \mathrm{~K}_{2} \mathrm{O} \mathrm{ha}{ }^{-1}\right)$ em relação à ausência do fornecimento de $\mathrm{K}$ (dose zero), sendo que essa dose não se diferenciou significativamente das demais, exceto os nutrientes $\mathrm{K}$ e $\mathrm{Cu}$ que tiveram seus conteúdos aumentados até a terceira dose de K. A ausência ou pouco efeito de doses crescentes de NPK no acúmulo de micronutrientes na parte aérea dessa espécie possivelmente ocorra em função da pouca exigência da espécie quanto a esses nutrientes na fase de muda (SILVA et al., 2007).

Deve-se levar em conta que, se a concentração no tecido da planta de um determinado elemento nutriente essencial cai abaixo do nível necessário para o crescimento ótimo, a planta é dita deficiente nesse elemento. Comparações entre as concentrações dos elementos essenciais (macros e micronutrientes) obtidas na espécie mogno no presente estudo foram feitas com as concentrações comumente encontradas em material vegetal e sugeridas como ideais, acima das quais a maioria das plantas não sofre limitações no crescimento (STOUT, 1961, citado por EPSTEIN, 2004). Os resultados encontrados mostraram que a maioria das concentrações desses nutrientes encontrava-se dentro dos limites aceitáveis, e aqueles que se encontravam abaixo tinham suas concentrações ideais satisfeitas a partir da adição da segunda dose dos nutrientes avaliados no presente estudo, o que possivelmente ratifica a ausência de resposta do mogno à adição de nutrientes na fase de mudas, como relatado anteriormente. 
Tabela 6. Conteúdo de nutrientes na parte aérea das mudas de mogno, em resposta a doses de potássio, aos 100 dias, em condição de casa de vegetação.

Table 6. Shoot nutrient contents of mahogany seedlings in response to potassium doses, after 100 days in greenhouse condition.

\begin{tabular}{|c|c|c|c|c|c|c|c|c|c|c|}
\hline \multirow{2}{*}{$\begin{array}{l}\text { Doses de } \mathrm{K}_{2} \mathrm{O} \\
\text { kg.ha' }^{-1}\end{array}$} & $\mathbf{N}$ & $\mathbf{P}$ & $\mathbf{K}$ & $\mathbf{C a}$ & Mg & $\mathbf{S}$ & B & $\mathbf{C u}$ & Mn & Zn \\
\hline & \multicolumn{5}{|c|}{--------g/unidade experimental--------- } & \multicolumn{5}{|c|}{-------mg/unidade experimental------- } \\
\hline 0 & $5,55 \mathrm{~b}$ & $0,16 \mathrm{~b}$ & $1,65 \mathrm{c}$ & $2,53 \mathrm{~b}$ & $0,78 \mathrm{~b}$ & $0,20 \mathrm{~b}$ & $9 \mathrm{~b}$ & $1 \mathrm{~b}$ & $4 \mathrm{~b}$ & $8 \mathrm{~b}$ \\
\hline 20 & $10,48 \mathrm{a}$ & $0,54 \mathrm{a}$ & $4,58 \mathrm{~b}$ & $5,18 \mathrm{a}$ & $1,50 \mathrm{a}$ & $0,48 \mathrm{a}$ & $19^{\mathrm{a}}$ & $1 b$ & $11 \mathrm{a}$ & $15 \mathrm{a}$ \\
\hline 40 & $10,16 \mathrm{a}$ & $0,42 a$ & $5,77 \mathrm{a}$ & $5,36 a$ & $1,47 \mathrm{a}$ & $0,52 \mathrm{a}$ & $19^{\mathrm{a}}$ & $2 a$ & $12 \mathrm{a}$ & $17 \mathrm{a}$ \\
\hline 60 & $11,19 \mathrm{a}$ & $0,47 \mathrm{a}$ & $8,27 \mathrm{a}$ & $5,93 \mathrm{a}$ & $1,62 \mathrm{a}$ & $0,56 \mathrm{a}$ & $25^{\mathrm{a}}$ & $3 a$ & $14 \mathrm{a}$ & $20 \mathrm{a}$ \\
\hline 80 & $10,74 \mathrm{a}$ & $0,47 a$ & $7,30 \mathrm{a}$ & $5,00 \mathrm{a}$ & $1,35 \mathrm{a}$ & $0,55 \mathrm{a}$ & $19^{\mathrm{a}}$ & $2 a$ & $11 \mathrm{a}$ & $14 \mathrm{a}$ \\
\hline 100 & $8,67 \mathrm{a}$ & $0,44 \mathrm{a}$ & $6,38 \mathrm{a}$ & $4,68 \mathrm{a}$ & $1,31 \mathrm{a}$ & $0,46 \mathrm{a}$ & $17^{\mathrm{a}}$ & $2 \mathrm{a}$ & $11 \mathrm{a}$ & $15 \mathrm{a}$ \\
\hline
\end{tabular}

Médias seguidas de mesmas letras nas colunas não diferem estatisticamente ao nível de $5 \%$.

Em relação ao índice de qualidade (IQD), que mede a relação entre os parâmetros de crescimento da planta, nota-se que o comportamento desses parâmetros em função da adição de NPK foi bastante específico e dependente do nutriente em particular, de modo que a adição de doses crescentes de $\mathrm{N}$ e P não proporcionou diferenças significativas do IQD (Tabela 7). Avaliando o crescimento e qualidade de mudas de Piptadenia gonoacantha (pau-jacaré) em resposta a fontes e doses de N, Marques et al. (2009) verificaram ausência de significância para a combinação dos fatores fontes e doses. Por outro lado, houve efeito isolado dos mesmos, de modo que a aplicação de sulfato de amônio produziu o maior índice e, consequentemente, mudas de melhor qualidade. No entanto, deve ser levado em conta que o pau-jacaré é uma espécie classificada como pioneira, o que possivelmente aumenta a probabilidade de resposta à adição de nutrientes e consequentemente uma maior importância da nutrição na qualidade das mudas.

Tabela 7. Índice de qualidade de Dickson (IQD) em mudas de mogno, em função da adição de doses crescentes de N, P e K, aos 100 dias, em condição de casa de vegetação.

Table 7. Dickson quality índex (IQD) in mahogany seedlings in response to nitrogen, phosphorus and potassium doses, after 100 days in greenhouse condition.

\begin{tabular}{lcc}
\hline Nutrientes & Doses $\left(\mathbf{k g}^{\left.-\mathbf{h} \mathbf{-}^{-1}\right)}\right.$ & IQD \\
\hline & 0 & $0,9 \mathrm{a}$ \\
$\mathrm{N}$ & 50 & $1,2 \mathrm{a}$ \\
& 100 & $0,9 \mathrm{a}$ \\
& 125 & $0,9 \mathrm{a}$ \\
& 150 & $0,9 \mathrm{a}$ \\
& 200 & $0,9 \mathrm{a}$ \\
\hline & 0 & $0,4 \mathrm{a}$ \\
$\mathrm{P}$ & 75 & $0,3 \mathrm{a}$ \\
& 150 & $0,3 \mathrm{a}$ \\
& 225 & $0,4 \mathrm{a}$ \\
& 300 & $0,3 \mathrm{a}$ \\
& 400 & $0,4 \mathrm{a}$ \\
$\mathrm{K}$ & 0 & $0,5 \mathrm{~b}$ \\
& 40 & $0,8 \mathrm{a}$ \\
& 80 & $0,7 \mathrm{a}$ \\
& 120 & $0,5 \mathrm{~b}$ \\
& 160 & $0,9 \mathrm{a}$ \\
& 200 & $0,8 \mathrm{a}$ \\
\hline
\end{tabular}

Médias seguidas de mesmas letras não diferem estatisticamente ao nível de 5\%.

Quanto ao fornecimento do nutriente $\mathrm{K}$, no presente estudo, verifica-se que, apesar de significativo, o efeito dele nos valores do IQD não foi muito consistente, uma vez que os menores valores de IQD foram observados tanto na ausência do fornecimento (dose zero) quanto na dose $120 \mathrm{~kg}^{-h^{-1} \mathrm{~K}}$ (Tabela 7). De modo geral, apesar de não ter se diferenciado significativamente, os valores do IQD 
variam de 0,9 a 1,2 para $\mathrm{N}$ e 0,3 a 0,4 para $\mathrm{P}$, enquanto no caso do $\mathrm{K}$ nota-se que, apesar de serem significativos, os valores foram menores que os observados para o N. Levando-se em conta que o valor mínimo do IQD para mudas de boa qualidade é 0,20 (GOMES, 2001), os resultados indicam que, apesar da pouca ou da ausência de resposta da espécie quanto a NPK, essas mudas apresentam bons índices de qualidade, pois os valores de IQD ficaram sempre acima de 0,2 .

No caso do presente estudo, parece haver uma estreita relação entre o comportamento dos parâmetros de crescimento de forma isolada e na forma combinada (IQD), pois em ambos os casos houve pouca ou ausência de influência do fornecimento de NPK. No caso da produção de matéria seca, essa estreita relação, possivelmente, se deve ao fato de ela ter grande influência no valor final desse índice. Segundo Chaves e Paiva (2004), na avaliação da qualidade das mudas se recomenda a utilização de vários parâmetros, uma vez que a determinação de índices isolados pode não avaliar adequadamente a qualidade delas, a exemplo de mudas muito altas, que geralmente têm se mostrado mais fracas, comprometendo o seu desenvolvimento no campo. Além disso, esses índices podem variar de acordo com a espécie, dentro da mesma espécie e de acordo com as características ambientais do local em que ela se encontra, como o estado de fertilidade do substrato. De modo geral, os resultados obtidos mostram que o manejo dessas mudas com baixo fornecimento de NPK já permite que elas superem o valor mínimo do IQD desejado, indicando que o aumento de doses crescentes desses nutrientes não influencia na qualidade da muda, principalmente no caso de $\mathrm{N}$ e $\mathrm{P}$.

A ausência ou baixo potencial de resposta do mogno na fase de mudas mostra que essa espécie apresenta um comportamento bastante diferente em relação à maioria das espécies mais cultivadas, pois elas têm sido consideradas bastante dependentes da adubação com os macronutrientes NPK. Tal observação é confirmada tanto para o comportamento dos teores e conteúdos desses nutrientes na parte aérea quanto no tecido radicular, além do comportamento das variáveis altura, diâmetro, MSPA, MSR e $\mathrm{R} / \mathrm{PA}$, indicando uma baixa eficiência de utilização, sendo também menos responsivas ao fornecimento de nutrientes, mesmo quando cultivada em solo que apresenta baixo $\mathrm{pH}$, alta saturação por alumínio e baixa fertilidade, como o que utilizado no presente estudo (Tabela 1).

No entanto, deve ser levado em conta que esse comportamento tem sido observado principalmente por trabalhos conduzidos em condições de casa de vegetação, sendo que, para confirmar tal premissa, mais trabalhos devem ser conduzidos, principalmente em condições de campo, o que permitiria uma inferência com maior consistência quanto ao manejo da adubação dessa espécie.

\section{CONCLUSÕES}

- O desenvolvimento das mudas de mogno é pouco influenciado pelo fornecimento de doses crescentes dos nutrientes nitrogênio, fósforo e potássio, indicando uma baixa exigência nutricional da espécie na fase inicial de crescimento.

- Entre os nutrientes avaliados, a espécie parece ligeiramente mais exigente quanto ao potássio em relação aos demais. No entanto, a demanda da planta já seria satisfeita com uma pequena adição do respectivo nutriente.

- A adubação com nitrogênio e fósforo não promove melhora na qualidade das mudas de mogno.

\section{REFERÊNCIAS}

BERNADINO, D. C. S.; PAIVA, H. N. de; NEVES, J. C. L.; GOMES, J. M.; MARQUES, V. B. Crescimento e qualidade de mudas de Anadenanthera macrocarpa (Benth.) Brenan em resposta à saturação por bases do substrato. Árvore, Viçosa, v. 29, n. 6, p. 863 - 870, 2005.

CAMARGOS, S. L.; MURAOKA, T.; FERNANDES, S. A. P.; SALVADOR, J. O. Diagnose nutricional em mudas de castanheira-do-brasil. Agricultura Tropical, Cuiabá, v. 6, n. 1, p. 81 - 96, 2002.

CARNEIRO, L. F.; FURTINI NETO, A. E.; RESENDE, A. V. de; CURI, N.; SANTOS, J. Z. L.; LAGO, F. J. do. Fontes, doses e modos de aplicação de fósforo na interação fósforo-zinco em milho. Ciência e Agrotecnologia, Lavras, v. 32, n. 4, p. 1133 - 1141, 2008. 
CHAVES, A. S.; PAIVA, H. N. Influência de diferentes períodos de sombreamento sobre a qualidade de fedegoso (Senna macranthera (Collad.) Irwin et Barn). Scientia Forestalis, n. 65, p. 22 - 29, 2004.

COMISSÃO DE FERTILIDADE DO SOLO DO ESTADO DE MINAS GERAIS (CFSMG). Recomendação para o uso de corretivos e fertilizantes em Minas Gerais: $5^{\underline{a}}$ Aproximação. Viçosa, 1999. $359 \mathrm{p}$.

CORDEIRO, Y. E. M.; PINHEIRO, H. A.; SANTOS FILHO, B. G.; CORRÊA, S. S.; SILVA, J. R. R.; DIAS FILHO, M. B. Physiological and morphological responses of young mahogany (Swietenia macrophylla King) plants to drought. Forest Ecology and Management, v. 258, p. 1449 - 1455, 2009.

CRAVO, M. S.; SMITH, T. J. Manejo sustentado da fertilidade de um Latossolo da Amazônia Central sob cultivos sucessivos. In: REUNIẪO BRASILEIRA DE FERTILIDADE DO SOLO E NUTRIÇÃO DE PLANTAS, 20., 1992, Piracicaba. Anais da... Piracicaba , 1992. p. 608 - 616.

DIAS, E. S.; KALIFE, C.; MENEUCCI, Z. R. H.; SOUZA, P. R. de. Produção de mudas de espécies florestais nativas. Campo Grande: Ed. UFMS, 2006. 59 p.

DICKSON, A.; LEAF, A. L.; HOSNER, J. F. Quality appraisal of white spruce and white pine seedling stock in nurseries. Forest Chronicle, v. 36, p. 10 - 13, 1960.

EPSTEIN, E.; BLOOM, A. Nutrição mineral de plantas: princípios e perspectivas. 2. ed. Londrina: Ed. Planta, 2004. $401 \mathrm{p}$.

FALCÃO, N. P. S.; SILVA, J. R. A. da. Características de adsorção de fósforo em alguns solos da Amazônia Central. Acta Amazônica, v. 34, n. 3, p. 337 - 342, 2004.

FREIRE, J. C.; RIBEIRO, M. A. V.; BAHIA, V. G.; LOPES, A. S.; AQUINO, L. E. Resposta do milho cultivado em casa de vegetação a níveis de água em solos da região de Lavras, MG. Revista Brasileira de Ciência do Solo, v. 4, n. 5, p. 5 - 8, 1980.

GOMES, J. M. Parâmetros morfológicos na avaliação da qualidade de mudas de Eucalyptus grandis, produzidas em diferentes tamanhos de tubete e de dosagens de N-P-K. 166 p. Tese (Doutorado em Ciência Florestal) - Universidade Federal de Viçosa, Viçosa, 2001.

GONÇALVES, J. L. M.; FREIXÊDAS, V. M.; KAGEYAMA, P. Y.; GONÇALVES, J. C.; DIAS, J. H. Produção de biomassa e sistema radicular de espécies de diferentes estágios sucessionais. Revista do Instituto Florestal, v. 4, p. 363 - 367, 1992.

LAMBERS, H.; POORTER, H. Inherent variations in growth rate between higher plants: a search for physiological causes and ecological consequences. Advances in Ecological Research, v. 23, p. 188 $261,1992$.

LAMPRECHT, H. Silvicultura nos trópicos: ecossistemas florestais e respectivas espécies arbóreas possibilidade e métodos de aproveitamento sustentado. Tradução: Guilherme de Almeida-Sedas e Gilberto Calcagnotto. Eschborn: GTZ, 1990. 343 p.

LIMA, H. N.; VALE, F. R.; SIQUEIRA, J. O.; CURI, N. Crescimento inicial a campo de sete espécies arbóreas nativas em resposta à adubação mineral com NPK. Revista Ciência e Agrotecnologia, v. 21, n. 1, p. 189 - 195, 1997.

LORENZI, H. Árvores brasileiras: manual de identificação e cultivo de plantas arbóreas nativas do Brasil. 2. ed. Nova Odessa: Instituto Plantarum, v. 1, 1998. 352 p.

MAFFEIS, A. R.; SILVEIRA, R. L. V. de A.; BRITO, J. O. Reflexos das deficiências de macronutrientes e boro no crescimento de plantas, produção e qualidade de óleo essencial em Eucalyptus citriodora. Scientia Forestalis, Piracicaba, v. 57, p. 87 - 98, 2000.

Malavolta, E.; VITTI, G. C.; OLIVEIRA, S. A. Avaliação do estado nutricional das plantas: princípios e aplicações. 2. ed. Piracicaba: Potafos, 1997. 319 p. 
MARQUES, L. S.; PAIVA, H. N. de; NEVES, J. C. L.; GOMES, J. M.; SOUZA, P. H. de. Crescimento de mudas de jacaré (Piptadenia gonoacantha J. F. Macbr.) em diferentes tipos de solos e fontes e doses de nitrogênio. Árvore, Viçosa, v. 33, n. 1, p. 81 - 92, 2009.

RESENDE, A. V. de; FURTINI NETO, A. E.; MUNIZ, J. A.; CURI, N.; FAQUIN, V. Crescimento inicial de espécies florestais de diferentes grupos sucessionais em resposta a doses de fósforo. Pesquisa Agropecuária Brasileira, Brasília, v. 34, n. 11, p. 2071 - 2081, 1999.

SANTOS, J. Z. L.; RESENDE, A. V. de; FURTINI NETO, A. E.; CORTE, E. F. Crescimento, acúmulo de fósforo e frações fosfatadas em mudas de sete espécies arbóreas nativas. Árvore, Viçosa, v. 32, n. 5, p. $799-807,2008$.

SANTOS, R. A.; TUCCI, C. A. F.; HARA, F. A. S.; SILVA, W. G. da. Adubação fosfatada para a produção de mudas de mogno (Swietenia macrophylla King). Acta Amazônica, v. 38, n. 3, p. 453 - 458, 2008.

SILVA, A. R. M. da; TUCCI, C. A. F.; LIMA, H. N.; FIGUEIREDO, A. F. Doses crescentes de corretivo na formação de mudas de mogno (Swietenia macrophylla King). Acta Amazônica, v. 37, n. 2, p. 195 200, 2007.

SILVA, I. R. da; FURTINI NETO, A. E.; CURI, VALE, F. R. do. Crescimento inicial de quatorze espécies florestais nativas em resposta à adubação potássica. Pesquisa Agropecuária Brasileira, Brasília, v. 32, n. 2, p. 205 - 212, 1997.

SISTEMA PARA ANÁLISES ESTATÍSTICAS (SAEG). Versão 9.1. Viçosa: Fundação Arthur Bernardes; UFV, 2007.

TUCCI, C. A. F.; LIMA, H. N.; LESSA, J. F. Adubação nitrogenada na produção de mudas de mogno (Swietenia macrophylla King). Acta Amazônica, v. 39, n. 2, p. 289 - 294, 2009.

WALlAU, R. L. R. de; BORGES, A. R.; ALMEIDA, D. R. de; CAMARGOS, S. L. Sintomas de deficiências nutricionais em mudas de mogno cultivadas em solução nutritiva. Cerne, Lavras, v. 14, n. 4, p. $304-310,2008$. 